\title{
Stability of dual solutions of mass transfer on a continuous flat plate moving in parallel or reversely to a free stream in the presence of a chemical reaction with second order slip
}

\begin{abstract}
The present paper investigates the steady viscous flow and mass transfer on a moving plate in parallel or reversely to a free stream in the presence of chemical reaction using a second order slip flow model. The governing partial differential equations are transformed into nonlinear ordinary differential equations by using appropriate similarity transformations, which are then solved numerically using shooting method for different values of selected parameters. We found that dual solutions exist for a certain range of the velocity ratio parameter. By using bvp4c solver in Matlab software, a stability analysis is performed to show that the first solutions are stable and physically relevant, while the second solutions are unstable and not physically relevant. The effects of reaction rate parameter, Schmidt number, first order slip parameter and second order slip parameter on the skin friction coefficient, mass transfer from the surface of the plate, dimensionless velocity and concentration profiles are figured out graphically and discussed. These results reveal that the second order slip flow model is necessary to give better prediction to the flow behavior.
\end{abstract}

Keyword: Mass transfer; Chemical reaction; Second order slip flow; Stability analysis 